\title{
Factors of Cell Degeneration or Death caused by Mutant Tau Protein
}

\author{
O Mikiko ${ }^{1 *}$ \\ ${ }^{1}$ Dalian Maple Leaf International High School, Liaoning 116650, China
}

\begin{abstract}
Tau protein is a microtubule associated protein mainly expressed in neurons. Under pathological conditions, Tau protein is abnormally hyperphosphorylated and separated from microtubules. Abnormal Tau aggregates form nerve fiber tangles, which are insoluble aggregates in the brain. It is due to the microtubule rupture caused by Tau protein dysfunction and it is associated with neurofibrillar degeneration in Alzheimer's disease.This paper studies several reports and research on the structure and function of Tau protein, the role of Tau protein in pathological diseases and its relationship with neurodegenerative diseases. This paper concludes that Tau protein has undergone abnormal modification and aggregation in many neurodegenerative diseases, but the specific type of Tau protein that causes neurotoxicity, as well as the pathogenesis of its phosphorylation and functional injury inducing nerve apoptosis, are still not fully understood. Various abnormal modifications of Tau protein occur under pathological conditions, and fatal cascade events occur at different stages of neuron apoptosis. Therefore, the causes and effects of cytotoxicity mediated by Tau protein are very complicated. Different or even opposite conclusions are sometimes drawn in Tau protein-mediated neurodegeneration studies. This may be due to differences in Tau protein type, gene mutation and protein expression level.
\end{abstract}

\section{Introduction}

Tau protein is a microtubule-associated protein, which is mainly expressed in neurons. Tau protein's normal function is to promote the assembly of tubules into microtubules and maintain the stability of the formed microtubules. It regulates the growth and development of nerve cells and plays an important role in the formation of the nervous system and axon transmission [1]. Under pathological conditions, Tau protein is abnormally hyperphosphorylated and separated from microtubules. Abnormal Tau aggregates form nerve fiber tangles, which are insoluble aggregates in the brain. It is caused by the breakdown of microtubules by dysfunctions of Tau protein. It is related to neurofibrillary degeneration of Alzheimer's disease [2]. This research paper is concerned with Tau's structure and functions, Tau protein in pathologic disease, and its relationship with neurodegenerative disease (Alzheimer's disease).

\section{Biological characteristics and structure of Tau protein}

\subsection{The structure of Tau protein}

The human Tau protein gene is located on the chromosome 17, which is over $100 \mathrm{KBP}$ in length and contains 15 exons. The six variants derived from different mRnas of the same gene contain 352-441 amino acid residues. They differ in that the $\mathrm{C}$-terminus has 3 or 4 microtubule-associated regions and the $\mathrm{N}$-terminus has 0,1 or 2 insertion sequences consisting of 29 amino acids Three of the six variants of Tau protein contain four microtubule-associated regions, and the other three lack a microtubule-associated region encoded by exon 10 , which contains another cysteine in Tau. The N-terminus consists of a region of acidic amino acids followed by a region of mostly basic amino acids. There is a proline-rich region (about $25 \%$ proline) upstream of the microtubule-associated region, and a basic amino acid region downstream. Tau protein in its natural state is mainly open and lose conformation, with only a small amount of alpha-helix and beta-sheet [3].

\subsection{The function of Tau protein}

Tau protein in a normal state can capture tubules and assemble them into microtubules. It can stabilize the formed microtubules. Microtubule affinity regulatory kinases regulate the transport function of microtubule by phosphorylating and modifying microtubule binding proteins (including Tau) so that they break away from the microtubule. Madelknow and others demonstrated with $\mathrm{CHO}$ cell lines that various microtubule-associated proteins have similar effects on microtubule transport. [4].

\footnotetext{
* Corresponding author: 2017011423@students.maoleleafedu.com
} 


\section{Tau protein in pathologic state}

\subsection{Tau abnormalities and neurodegenerative Tau disease}

Previous autopsies showed that there was a good parallel relationship between brain nerve fiber tangles and neuronal death, abnormal cell morphology and apoptosis. Therefore, NFTs was considered to be an important pathogenesis factor of neurodegenerative diseases such as $\mathrm{AD}$ and FTD, and the formation and influence factors of NFTs were discussed. Preliminary studies have shown that in pathological conditions (such as in $\mathrm{AD}$ ), various pathogenic factors lead to the imbalance of Tau kinase and phosphatase function through different pathological pathways, leading to the abnormally high level of Tau phosphorus. Acidification so that phosphate receptor residues are mostly phosphorylated; Thus, the dynamic balance of Tau protein binding with microtubules is disturbed, which leads to the abnormal increase of free Tau protein fragments. That finally leads to abnormal Tau aggregation, fibrosis and NFTs formation [5].

According to later studies, mutations at several sites on Tau exon 10 may affect the expression ratio of $3 R$ and $4 \mathrm{R}$ Tau isomers. It is characterized by $4 \mathrm{R}$ Tau overproduction. The $4 \mathrm{R}$ Tau protein has a greater affinity for microtubules than the $3 \mathrm{R}$ Tau protein [6]. Other researchers also believe that the Tau mutation makes the Tau protein more easily assembled into filaments, making it more prone to fibrosis [7].

\begin{tabular}{|l|l|}
\hline Tau Protein Marker & T08-07N \\
\hline Tau-316 Protein & T02-54N \\
\hline Tau-352 Protein & T03-54N \\
\hline Tau-381 Protein & T04-54N \\
\hline Tau-383 Protein & T05-54N \\
\hline Tau-410 Protein & T06-54N \\
\hline Tau-412 Protein & T07-54N \\
\hline Tau-441 Protein & T08-54N \\
\hline Tau-441 Protein & T08-54H \\
\hline Tau-441, Biotinylated & T08-54BN \\
\hline
\end{tabular}

Fig.1. Normal Tau Protein

\begin{tabular}{|l|l|}
\hline Tau-383 (N352H) Protein & T05-56N \\
\hline Tau-441 (A152T) Protein & T08-56VN \\
\hline Tau-441 (dK280) Protein & T08-52N \\
\hline Tau-441 (dN296) Protein & T08-52BN \\
\hline Tau-441 (G272V) Protein & T08-56DN \\
\hline Tau-441 (K257T) Protein & T08-56N \\
\hline Tau-441 (L266V) Protein & T08-56CN \\
\hline Tau-441 (N279K) Protein & T08-56EN \\
\hline Tau-441 (P301L) Protein & T08-56FN \\
\hline Tau-441 (P301S) Protein & T08-56GN \\
\hline Tau-441 (R406W) Protein & T08-56MN \\
\hline Tau-441 (S198A) Protein & T08-53BN \\
\hline Tau-441 (S198E) Protein & T08-53N \\
\hline Tau-441 (S199E) Protein & T08-53CN \\
\hline Tau-441 (S214A) Protein & T08-53DN \\
\hline Tau-441 (S305N) Protein & T08-56HN \\
\hline Tau-441 (S352L) Protein & T08-56KN \\
\hline Tau-441 (S404A) Protein & T08-53HN \\
\hline Tau-441 (S404E) Protein & T08-53GN \\
\hline Tau-441 (N337M) Protein & T08-56JN \\
\hline
\end{tabular}

Fig.2. Mutant Tau Protein

\subsection{The helical filament structure of Tau protein in pathological state}

Under pathological conditions, abnormal phosphorylation or mutation of Tau protein reduces its affinity with microtubules, which leading to increased intracellular free Tau protein concentration. High concentration of Tau protein provides a prerequisite for Tau protein aggregation to form helical filaments. Different diseases in the formation of the spiral wire form and Tau protein variants molecules is not the same. Nearly 125 years people using electron microscopy, 
X-ray diffraction, and Fourier transform infrared spectroscopy restructuring Tau protein in vitro formation of the spiral wire is studied. The spiral wire in nature, found in the spiral wire Tau protein formed a large number of Beta folding conformation. At present, the understanding of helical filaments formation process mainly comes from in vitro simulation experiment. In 1992, recombinant Tau protein was used for the first time to simulate the formation of an obscure helix with similar diameters and helical cycles to the natural helix. The formation of helical filaments was promoted by dimerization of two Tau molecules through disulphin bonds, while the formation of helical filaments was blocked by the mutation of cys322 to Ala [8]. After the formation of dimers, the process of aggregation into helical filaments was still very slow. Later, sGAGs and RNA were found to exist in helical filaments. Further studies have shown that these polyanions and many other polyanion compounds can accelerate the formation of helical filaments. It was found that after the formation of dimers, there was still a kinetic barrier in the presence of polyanions, which hindered the rapid formation of helical filaments [9]. Tau protein must first form some core bodies composed of 4-7 dimer units after the formation of dimer, in order to quickly form spiral filaments with the assistance of polyanions [8]. The mutation of Tau protein reduces its affinity with microtubules, leading to the increase of free Tau protein and further formation of nerve fiber tangles.

According to later studies, mutations at several sites on Tau exon 10 May affect the expression ratio of 3R and $4 \mathrm{R}$ Tau isomers. It is characterized by $4 \mathrm{R}$ Tau overproduction. The $4 \mathrm{R}$ Tau protein has a greater affinity for microtubules than the $3 \mathrm{R}$ Tau protein. Other researchers also believe that the Tau mutation makes the Tau protein more easily assembled into filaments, making it more prone to fibrosis.

\subsection{Abnormal Tau phosphorylation and nerve cell death}

Tau protein has $20 \sim 30$ phosphorylation sites, and its phosphorylation status is mainly determined by the relative activities of protein kinase and protein phosphatase. A large number of experiments showed that GSK-3B and CDK-5 were the two major kinases leading to hyperphosphorylation of Tau protein. In 2004, an experiment used the PKA agonist Forskolin to study Tau phosphorylation levels in adult rats. They believed that PKA phosphorylation of Ser at 214 could make Tau protein more prone to be phosphorylated by GSK-3b, and blocking Tau protein phosphorylation by PKA inhibitor could restore the spatial memory ability of rat [10]. In addition, another experiment found that in addition to kinases and phosphatases, Pinl, a protein isomerase, also plays an important regulatory role in Tau phosphorylation. The team found that Tau occurs in two different conformations after hyperphosphorylation. Pin1 catalyzes the conversion of Tau protein from one conformation to another. This change can make the phosphorylated Tau protein easily dephosphorylated by PP-2A [11].

Many factors can change the activity of kinase or phosphatase through a variety of signal transduction pathways, leading to Tau hyperphosphorylation. The hyperphosphorylation of Tau protein reduces the affinity between Tau protein and microtubules. On the one hand, Tau dissociates from microtubules, causing microtubules dysfunction and further leading to nerve cell death. On the other hand, the concentration of free Tau protein increases, providing conditions for further aggregation and formation of helical filaments. Hyperphosphorylated Tau protein reduces motor protein adsorption on microtubules and weakens the axonal transport capacity of cells. Glycosylation is related to the stability of helix structure, especially to the cycle maintenance of helix in helix. Simple deglycation treatment can not restore the biological activity of abnormal Tau protein but can increase the subsequent dephosphorylation [11].

However, in 2016, an Australian study suggested that Tau phosphorylation actually protects neurons from damage. Researchers believe beta-amyloid is toxic to neurons, but the first step in tau phosphorylation actually reduces this toxicity. Previously, scientists believed that the protein that forms plaques, beta-amyloid, causes a phosphorylation modification of tau that leads to cell death and, ultimately, Alzheimer's disease. Increased tau phosphorylation eventually causes the protein to form tangles. The results of this new study suggest that tau phosphorylation initially has a protective effect on neurons, and that amyloid beta attacks this protective function until it is lost. It is the level of toxicity at this stage that leads to neuronal destruction and cognitive deficits associated with Alzheimer's disease [12].

What this debunking study is trying to show is that there are mutations at specific sites where phosphorylation can actually be beneficial. But in general, abnormal tau phosphorylation does cause a lot of problems.

\subsection{Tau protein causes nerve cell death in other ways}

Studies have shown that non-enzymatic glycosylation of glucose and Tau protein can promote the formation of helical filaments. The glycosylated Tau protein molecules can be rearranged through Amadori to form stable glycosylated products. Its one-step oxidation can form advanced glycosylated end products, which are difficult to remove in vivo and have cytotoxicity. In 1999, Durany with others found advanced glycosylation end products in the brain lamella of Alzheimer's patients. They suggested that advanced glycosylation end products increase oxidative stress, leading to nerve cell death by triggering the production of free radicals [13].

In 2001, a study found that the helical filaments can combine with $\mathrm{Ca} 2+$ and $\mathrm{Fe} 2+$ to form a complex, thus obtaining the function of catalytic $\mathrm{H} 2 \mathrm{O} 2$-dependent diaminobenzidine oxidation. The phenomenon of promoting diethylenediamine oxidation reflects the production of ROS in vivo caused by spiral filament. 
They also found that glycosylation increased the binding level of the helical wires to the metal ions. The glycosylation of helical filaments and their combination with $\mathrm{Cu} 2+$ and $\mathrm{Fe} 2+$ catalyzed diethylenediamine oxidation can lead to nerve cell death [14].

In 2003, Kecks et al. studied the proteasome activity of patients with Alzheimer's disease and the control group, and found that the proteasome activity of patients with Alzheimer's disease was significantly reduced, and this decrease was not caused by a decrease in the amount of proteasome. The team found in vitro that helical filaments can degrade their activity by binding to proteasomes, and that the blocking effect is sufficient to cause nerve cell death. They thought Tau can directly lead to nerve cell death by reducing proteasome activity [15].

\section{Tau phosphorylation and Alzheimer's disease}

Tau phosphorylation in AD patients is 3-4 times higher than that in normal people. There are three types of tau proteins in the brain of patients with $\mathrm{AD}$, namely normal cytoplasmic Tau (C-Tau), hyperphosphorylated soluble Tau (ADP-Tau), and Tau proteins that aggregate into PHF (PHF-Tau). At present, 45 phosphorylation sites have been identified in PHF-Tau. They are mainly phosphorylated by threonine and serine residues, mostly concentrated in PRD and C-terminus regions of tau protein, and a few in MBD region. Under normal circumstances, most sites are not phosphorylated, but remain phosphorylated in the brain of $\mathrm{AD}$ patients. Phosphorylation of Tau protein in MBD is essential to regulate the stability of microtubules, especially phosphorylation at Ser262 and Ser356, which changes the conformation of tau protein and microtubules, leading to microtubules disintegration and cytoskeleton destruction [16].

Tau hyperphosphorylation is an important initial step in the degradation of AD brain neurons. Hyperphosphorylated Tau protein not only inhibits the pro-microtubule assembly activity, but also isolated the normal Tau protein, MAPl and MAP2 involved in microtubule assembly, further damaging the microtubule assembly, leading to neuronal dysfunction and degeneration. In the early stage of neuronal apoptosis, phosphorylation of many epitopes of Tau protein was significantly increased, leading to a decrease in tau's ability to bind to microtubules. However, the hyperphosphorylated Tau protein is dephosphorylated and degraded in the final stage of apoptosis, and its presence is transient [17]. Therefore, it is believed that Tau hyperphosphorylation coexists with neuronal apoptosis, and is not an independent event. Tau hyperphosphorylation is likely to be the initiating factor in the apoptosis process.

\section{Conclusion}

Neurofibrillary tangles are a pathological feature of a variety of nervous system diseases, of which Alzheimer's disease is the most common. Tau protein in many neurodegenerative diseases has undergone abnormal modification and aggregation, but the specific type of Tau protein that leads to neurotoxicity, as well as the pathogenesis of tau hyperphosphorylation and functional injury in inducing nerve apoptosis is still not fully understood. A variety of abnormal modifications of Tau protein occur in pathological conditions, and fatal cascade events occur in different stages of neuronal apoptosis. Therefore, the causes and effects of Tau protein-mediated cytotoxicity are very complex. In studies of Tau protein-mediated neurodegeneration, different or even opposite conclusions are sometimes drawn. This may be due to differences in Tau protein types, gene mutations and protein expression levels. Therefore, further research is needed to determine the function of Tau proteins and how they affect Tau aggregation, redistribution, and abnormal modification in the patient's brain. These studies will contribute to elucidate the specific mechanisms by which Tau mediated neuronal dysfunction and apoptosis and to interpret key events that occur in Tau mediated neurotoxicity. There are still some deficiencies in this paper, which need to be strengthened in terms of practicability and insufficient connection with clinical cases. In conclusion, further understanding of the initiation events of Tau protein-mediated neurodegeneration may provide theoretical basis for early treatment strategies for many neurodegenerative diseases, such as AD.

\section{Acknowledgement}

First and foremost, I would like to show my deepest gratitude to my teachers in my school and professor for my research project, who have provided me with valuable guidance in every stage of the writing of this thesis. Further, I would like to thank all my friends and roomates for their encouragement and support. Without all their enlightening instruction and impressive kindness, I could not have completed my thesis.

\section{References}

1. Binder, L I et al. The distribution of Tau in the mammalian central nervous system. The Journal of cell biology vol. 101,4 (1985): 1371-8. doi:10.1083/jcb.101.4.1371

2. Korgiopoulou, Christina. "Tau Post-Translational Modifications in Alzheimer's Disease: Diagnostic and Therapeutic Opportunities: StressMarq." 1 June 2020 ,

www.stressmarq.com/tau-post-translational-modific ations-alzheimers-disease-diagnostic-therapeutic-op portunities/?v=3e8d115eb4b3. 
3. Friedhoff, P et al. "Structure of tau protein and assembly into paired helical filaments." Biochimica et biophysica acta vol. 1502,1 (2000): 122-32. doi:10.1016/s0925-4439(00)00038-7

4. Mandelkow, Eva-Maria, et al. "MARK/PAR1 Kinase is a Regulator of Microtubule-Dependent Transport in Axons." Journal of Cell Biology vol. 167, no. 1, 2004, pp. 99-110., doi:10.1083/jcb.200401085.

5. Xu, Shaohua, et al. "Characterization of Tau Fibrillization in Vitro." Alzheimer's \&amp;

Dementia, vol. 6, no. 2, 2010, pp. 110-117., doi:10.1016/j.jalz.2009.06.002.

6. Künze, Georg, et al. "Binding of the Three-Repeat Domain of Tau to Phospholipid Membranes Induces an Aggregated-like State of the Protein." Biochimica Et Biophysica Acta (BBA) Biomembranes, vol. 1818, no. 9, 2012, pp. 2302-2313., doi:10.1016/j.bbamem.2012.03.019.

7. Goedert, Michel, and Ross Jakes. "Mutations Causing Neurodegenerative Tauopathies." Biochimica Et Biophysica Acta (BBA) - Molecular Basis of Disease, vol. 1739, no. 2-3, 2005, pp. 240-250., doi:10.1016/j.bbadis.2004.08.007.

8. Friedhoff, $\mathrm{P}$ et al. "A nucleated assembly mechanism of Alzheimer paired helical filaments."

Proceedings of the National Academy of Sciences of the United States of America vol. 95,26 (1998): 15712-7. doi:10.1073/pnas.95.26.15712

9. Friedhoff, $P$ et al. "Rapid assembly of Alzheimer-like paired helical filaments from microtubule-associated protein tau monitored by fluorescence in solution." Biochemistry vol. 37,28 (1998): 10223-30. doi:10.1021/bi980537d

10. Liu, Shi Jie, et al. "Tau Becomes a More Favorable Substrate for GSK-3 When It Is Prephosphorylated by PKA in Rat Brain." Journal of Biological Chemistry, vol. 279, no. 48, 2004, pp. 50078-50088., doi:10.1074/jbc.m406109200.

11. Lim, Jormay, and Kun Ping Lu. "Pinning down Phosphorylated Tau and Tauopathies." Biochimica Et Biophysica Acta (BBA) - Molecular Basis of Disease, vol. 1739, no. 2-3, 2005, pp. 311-322., doi:10.1016/j.bbadis.2004.10.003.

12. Ittner, Arne et al. "Site-specific phosphorylation of tau inhibits amyloid- $\beta$ toxicity in Alzheimer's mice." Science (New York, N.Y.) vol. 354,6314 (2016): 904-908. doi:10.1126/science.aah6205

13. Durany, N., et al. "Investigations on Oxidative Stress and Therapeutical Implications in Dementia." European Archives of Psychiatry and Clinical Neurosciences, vol. 249, no. S3, 1999, doi:10.1007/p100014177.

14. Sayre, Lawrence M., et al. "In Situ Oxidative Catalysis by Neurofibrillary Tangles and Senile Plaques in Alzheimer's Disease." Journal of Neurochemistry, vol. 74, no. 1, 2001, pp. 270-279., doi:10.1046/j.1471-4159.2000.0740270.x.
15. Keck, Susi, et al. "Proteasome Inhibition by Paired Helical Filament-Tau in Brains of Patients with Alzheimer's Disease." Journal of Neurochemistry, vol. 85, no. 1, 2003, pp. 115-122., doi:10.1046/j.1471-4159.2003.01642.x.

16. Hanger, Diane P et al. "Tau phosphorylation: the therapeutic challenge for neurodegenerative disease." Trends in molecular medicine vol. 15,3 (2009): 112-9. doi:10.1016/j.molmed.2009.01.003

17. Castellani, Rudy J et al. "Phosphorylated tau: toxic, protective, or none of the above." Journal of Alzheimer's disease : JAD vol. 14,4 (2008): 377-83. doi:10.3233/jad-2008-14404. 